\title{
The Role of Identified Neurotransmitter Systems in the Response of Insular Cortex to Unfamiliar Taste: Activation of ERK1-2 and Formation of a Memory Trace
}

\author{
Diego E. Berman, Shoshi Hazvi, Victor Neduva, and Yadin Dudai \\ Department of Neurobiology, The Weizmann Institute of Science, Rehovot 76100, Israel
}

In the behaving rat, the consumption of an unfamiliar taste activates the extracellular signal-regulated kinase 1-2 (ERK1-2) in the insular cortex, which contains the taste cortex. In contrast, consumption of a familiar taste has no effect. Furthermore, activation of ERK1-2, culminating in modulation of gene expression, is obligatory for the encoding of long-term, but not short-term, memory of the new taste (Berman et al., 1998). Which neurotransmitter and neuromodulatory systems are involved in the activation of ERK1-2 by the unfamiliar taste and in the long-term encoding of the new taste information? Here we show, by the use of local microinjections of pharmacological agents to the insular cortex in the behaving rat, that multiple neurotransmitters and neuromodulators are required for encoding of taste memory in cortex. However, these systems vary in the specificity of their role in memory acquisition and in their contribution to the activation of ERK1-2. NMDA receptors, metabotropic glutamate receptors, muscarinic, and $\beta$-adrenergic and dopaminergic receptors, all contribute to the acquisition of the new taste memory but not to its retrieval. Among these, only NMDA and muscarinic receptors specifically mediate taste-dependent activation of ERK1-2, whereas the $\beta$-adrenergic function is independent of ERK1-2, and dopaminergic receptors regulate also the basal level of ERK1-2 activation. The data are discussed in the context of postulated novelty detection circuits in the central taste system.

Key words: memory; novelty; taste; acetylcholine; glutamate; MAPK
Consumption of even a small amount of an unfamiliar tastant is a salient experience that may suffice to imprint a long-term memory of the new taste and its hedonic valence (Bures et al., 1998). This single-trial learning capability has a clear phylogenetic advantage, e.g., it ensures that poisons, if survived, are never approached again. We attempt to elucidate how such efficient learning and robust memory are implemented in the rat brain. A key role in the detection of taste novelty and the encoding of taste memory is played by the central gustatory area in the insular cortex (IC) (Kiefer and Braun, 1977; Rosenblum et al., 1993, 1997; Schafe and Bernstein, 1998). We have identified previously multiple molecular mechanisms in the IC that are specifically turned on by an unfamiliar taste but not by a familiar one (Rosenblum et al., 1997; Berman et al., 1998). One of these mechanisms involves differential activation of mitogen-activated protein kinases (MAPKs), specifically the extracellular signal-regulated kinases 1-2 (ERK1-2) (Berman et al., 1998). Activation of ERK1-2 develops within half an hour after consumption of a new taste and triggers modulation of gene expression that subserves consolidation of memory in the IC (Berman et al., 1998). Reaction to taste in the behaving organism can be detected within seconds or even less (Halpern and Tapper, 1971). It is hence evident that the activation of ERK1-2 in the IC is triggered by earlier neuronal events that may also control immediate behavior. Furthermore, this activation is not induced merely by perception of the sensory attributes of the taste per se, because a familiar taste has no effect; additional input to cortex that encodes "unfamiliarity" is required. How is this done? A likely possibility is that certain neuromodulators, e.g., acetylcholine (Naor and Dudai, 1996; Rosenblum et al., 1996; Gutierrez et al., 1999), are recruited by the mismatch between the perceived and the familiar, instruct the IC that some action is needed to react to

\footnotetext{
Received Feb. 15, 2000; revised June 23, 2000; accepted June 26, 2000.

We thank Ehud Ahissar, Amir Bahar, and Roni Seger for discussions, and the Carl and Micaela Einhorn-Dominic Center for Brain Research for support.

Correspondence should be addressed to Dr. Yadin Dudai, Department of Neurobiology and Institute for Brain Research, The Weizmann Institute of Science, Rehovot 76100, Israel. E-mail: yadin.dudai@weizmann.ac.il.

Copyright (C) 2000 Society for Neuroscience $0270-6474 / 00 / 207017-07 \$ 15.00 / 0$
}

the new situation on the one hand, and register it in memory on the other.

In this study, we have investigated the effect of microinjection into the IC of antagonists and agonists of identified neurotransmitters and neuromodulators on the activation of ERK1-2 by unfamiliar taste and on the encoding of taste memory. Activation of ERK1-2 was selected as a molecular correlate of memory formation because it leads to activation of the transcription factor Elk-1 in the IC, and its blockade in the IC prevents taste memory (Berman et al., 1998). We report here that, whereas formation of taste memory in the IC can be disrupted by perturbation of multiple types of receptors for neurotransmitters, only a subgroup of these receptor types is differentially required in the acquisition of memory but not in its retrieval. Furthermore, only glutamatergic [NMDA receptor (NMDAR)-mediated] and cholinergic inputs function specifically in novelty-dependent activation of ERK1-2 in the IC. Our data also suggest that the basal activity level of ERK1-2 in the IC is maintained by a balance between glutamatergic, dopaminergic, and GABAergic inputs.

\section{MATERIALS AND METHODS}

Animals. Male Wistar rats ( $\sim 60$ d old, $250-300 \mathrm{gm})$ were used. They were caged individually at $22 \pm 2{ }^{\circ} \mathrm{C}$ in a $12 \mathrm{hr}$ light/dark cycle.

Reagents. Scopolamine, propranolol, bicuculline, and $(R, S)$ - $\alpha$-methyl-4carboxyphenylglycine (MCPG), monoclonal anti-diphospho-ERK (dpERK) (Thr183/Tyr185, MAPK-YT), anti-ERK antibodies, and goat anti-mouse $(\mathrm{IgG})$ peroxidase conjugate were from Sigma (St. Louis, MO). D,L-2-amino-5-phosphonovaleric acid (APV), muscimol, and $R(+)-7$ chloro-8-hydroxy-3-methyl-1-phenyl-2,3,4,5-tetrahydro-1H-3-benzazepine hydrochloride [SCH $23390(\mathrm{SCH})]$ were from Research Biochemicals (Natick, MA). 2,3-dioxo-6-nitro-1,2,3,4-tetrahydrobenzo[f]quinoxaline-7sulfonamide disodium (NBQX) was from Tocris Cookson (Bristol, UK). Horseradish peroxidase (HRP)-linked protein A and the enhanced chemiluminescence (ECL) kit were from Amersham Pharmacia Biotech (Buckingshamshire, UK). All other chemicals were of analytical grade or the highest grade available.

Behavioral procedures. We used two types of taste learning situations: incidental (Thorndike and Rock, 1934) and associative. The associative learning paradigm was conditioned taste aversion (CTA) (Revusky and Garcia, 1970; Bures et al., 1988, 1998). In CTA, organisms learn to avoid a novel taste if its ingestion is followed by transient malaise. We used the procedure described by Rosenblum et al. (1993, 1997). Unless indicated otherwise, saccharin was used as the unfamiliar taste, and $\mathrm{LiCl}(0.15 \mathrm{M}, 2 \%$ body weight, i.p.) was used as the malaise-inducing agent. The rats were 
water deprived for $24 \mathrm{hr}$ and then pretrained for $3 \mathrm{~d}$ to get their daily water ration once a day for $10 \mathrm{~min}$ from a pipette containing $10 \mathrm{ml}$ of water. On day 4 , they were allowed to drink the saccharin solution instead of water for $10 \mathrm{~min}$. Forty minutes after the offset of the drinking period, they were injected with $\mathrm{LiCl}$ intraperitoneally. Under these conditions, $3 \mathrm{~d}$ after training, the conditioned rats preferred water to saccharin at a ratio of 9:1 in a multiple choice test situation (Berman et al., 1998). The conditioned aversion is presented below as aversion index, defined as [milliliters of water $/($ milliliters of water + milliliters of saccharin $) \times 100]$, consumed in the test; hence, 50 indicates equal preference. In the "incidental learning" paradigm, rats were first water deprived for $24 \mathrm{hr}$ and then pretrained for $3 \mathrm{~d}$ to get their water ration from pipettes during $10 \mathrm{~min}$ per day, as above. On day 4, the animals were exposed for $10 \mathrm{~min}$ to the unfamiliar taste, sodium saccharin 0.1\% w/v (Rosenblum et al., 1993; Berman et al., 1998). The rats were then subjected to either a molecular or a behavioral analysis. For the molecular analysis, they were killed at the indicated time after taste consumption, and their IC was removed and processed for determination of the effect of the incidental exposure to the new taste on the activation of ERK1-2 (see below). For the behavioral analysis, the rats were subjected to CTA training $3 \mathrm{~d}$ later to determine the behavioral manifestation of the incidental training. Under this protocol, the incidental learning situation became the conditioned stimulus (CS) preexposure phase in a latent inhibition (LI) experiment (Lubow, 1989). In LI, preexposure to a sensory stimulus attenuates the effectiveness of that same stimulus to serve as a conditioned stimulus in subsequent learning. Indeed, the exposure of rats to an unfamiliar taste several days before CTA training significantly reduces the acquired aversion to that same taste (Rosenblum et al., 1997). In other words, the behavioral consequences of the incidental taste learning situation and their pharmacological modulation were hence determined in a protocol of LI of CTA (LI+CTA), in which CTA training was used as a probe for the efficacy of the incidental learning that has taken place a few days earlier.

Surgery and microinjection. Rats were anesthetized with $4.8 \mathrm{ml} / \mathrm{kg} \mathrm{Eq}-$ uithesin $\left(2.12 \% \mathrm{w} / \mathrm{v} \mathrm{MgSO}_{4}, 10 \% \mathrm{v} / \mathrm{v}\right.$ ethanol, $39.1 \% \mathrm{v} / \mathrm{v}$ propylene glycol, $0.98 \% \mathrm{w} / \mathrm{v}$ sodium pentobarbitone, and $4.2 \% \mathrm{w} / \mathrm{v}$ chloral hydrate), restrained in a stereotactic apparatus (David Kopf Instruments, Tujunga, $\mathrm{CA})$, and implanted bilaterally with stainless steel guide cannula (23 gauge, thin wall) aimed $1.0 \mathrm{~mm}$ above the gustatory neocortex [anteroposterior, $+1.2 \mathrm{~mm}$ relative to bregma; lateral, $\pm 5.5 \mathrm{~mm}$; ventral, $5.5 \mathrm{~mm}$ (Paxinos and Watson, 1986)]. The cannula were positioned in place with acrylic dental cement and secured by two skull screws. A stylus was placed in the guide cannula to prevent clogging. Animals were allowed 1 week to recuperate before being subjected to experimental manipulations. The stylus was removed from the guide cannula, and a 28 gauge injection cannula, extending $1.0 \mathrm{~mm}$ from the tip of the guide cannula, was inserted. The injection cannula were connected via PE20 tubing to a Hamilton microsyringe driven by a microinf usion pump (CMA/100; Carnegie Medicin, Stockholm, Sweden). Microinjection was performed bilaterally in a 1 $\mu \mathrm{l}$ volume per hemisphere delivered over $1 \mathrm{~min}$. The injection cannula was left in position before withdrawal for an additional $1 \mathrm{~min}$ to minimize dragging of the injected liquid along the injection tract. The sphere of diffusion of the injectate was estimated in a set of control animals microinjected with $1 \mu \mathrm{l}$ of India ink (Fig. 1) and found to overlap the gustatory area (Kosar et al., 1986). Drugs were dissolved in physiological saline or in artificial CSF (ACSF), and the appropriate vehicle was used as control. The particular drug dosages used for microinjection were selected on the basis of previous in vivo studies (Quillfeldt et al., 1994; Riedel et al., 1994; Naor and Dudai, 1996; Riedel, 1996; Rosenblum et al., 1997). In the case of MCPG, we have explored in preliminary experiments a dose range of 5-50 $\mu \mathrm{g}$ and selected the higher dose for further investigation.

Homogenization. Rats were decapitated $30 \mathrm{~min}$ after the completion of the exposure to the unfamiliar taste. The insular cortex containing the gustatory cortex was dissected out as described previously (Rosenblum et al., 1997). The tissue was immediately homogenized in a glass-Teflon homogenizer in $200 \mu \mathrm{l}$ of SDS sample buffer (10\% glycerol, $5 \%$ $\beta$-mercaptoethanol, and $2.3 \%$ SDS, in $62.5 \mathrm{~mm}$ Tris- $\mathrm{HCl}, \mathrm{pH} 6.8)$ and boiled for $5 \mathrm{~min}$. Samples were immediately stored at $-20^{\circ} \mathrm{C}$ until further usage.

Western blot analysis. Aliquots in SDS sample buffer were subjected to SDS-PAGE (8\% acrylamide) (Laemmli, 1970) and Western blot analysis (Burnette, 1981). The amount of protein in each sample was always determined before loading, and the same amount of protein $(60-100 \mu \mathrm{g})$ was loaded in each lane. After the run, the lanes were also compared by Ponceau staining. After blocking with $1 \%$ BSA for $1 \mathrm{hr}$ at room temperature, the blot was reacted overnight at $4^{\circ} \mathrm{C}$ or for $2 \mathrm{hr}$ at room temperature with the primary antibody and then incubated for $1 \mathrm{hr}$ at room temperature with HRP-linked protein A or with goat anti-mouse HRP-linked antibody before exposure to the ECL substrate. The antibodies directed against the phosphorylated ERK1-2 were usually applied first (dpERK, 1:30000). The blots were stripped in $0.9 \% \mathrm{w} / \mathrm{v} \mathrm{NaCl}, 10 \mathrm{~mm}$ Tris-HCl, $0.05 \% \mathrm{v} / \mathrm{v}$ Tween 20 , and $2 \% \mathrm{w} / \mathrm{v}$ SDS, $\mathrm{pH} 7.6$, four times for $10 \mathrm{~min}$ each at room temperature under vigorous shaking. The blots were then rinsed three times for $10 \mathrm{~min}$ in washing buffer (same stripping buffer without SDS) blocked for $1.5 \mathrm{hr}$ with $1 \% \mathrm{BSA}$, and incubated with the antibody antiERK1-2 (total ERK1-2, 1:2000). The efficacy of the stripping step was assessed by omitting the first antibody and verifying the lack of signals on

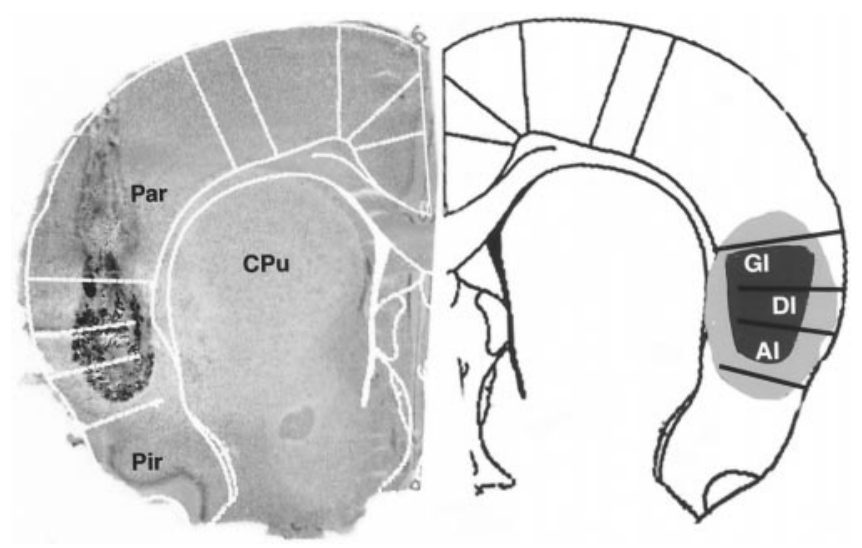

Figure 1. Left, A Nissl-stained frozen section of the rat brain [coronal cut, $+1.2 \mathrm{~mm}$ relative to bregma (Paxinos and Watson; 1986)] depicting the sphere of diffusion of $1 \mu \mathrm{l}$ of India ink microinjected into the insular cortex as detailed in Materials and Methods. Right, A scheme of the corresponding contralateral hemisphere depicting the superimposed sphere of diffusion in a group of 10 rats ( gray) and the estimated "median" obtained by superimposing the five smallest dye diffusion spheres (black). GI, Granular insular cortex; $D I$, dysgranular insular cortex; $A I$, agranular insular cortex; $C P u$, caudate-putamen; Par, parietal cortex; Pir, piriform cortex.

the blot. Quantification was performed using a computerized densitometer and image analyzer (Molecular Dynamics, Sunnyvale, CA).

Statistics. Differences among the groups were determined using one-way ANOVA. For paired comparisons, Scheffe contrast tests were used with an $\alpha$ level of 0.05 .

\section{RESULTS}

Perturbation of function of glutamatergic, cholinergic, $\boldsymbol{\beta}$-adrenergic, dopaminergic, or GABAergic receptors in the IC shortly before training impaired the encoding of taste memory

We have microinjected into the IC ligands that are specific for identified neurotransmitter and neuromodulatory systems shortly before the first exposure of rats to a novel taste solution and tested the effect on long-term taste memory. We have targeted the glutamatergic, cholinergic, dopaminergic, and GABAergic systems, because they were shown previously to function in the insular cortex (Lopez-Garcia et al., 1990; Otawa et al., 1995). The $\beta$-adrenergic system was also probed because of its presumed role in learning (Cahill et al., 1994), including in taste paradigms (Mohammed et al., 1986). Two types of complementary behavioral protocols were used. In the first (incidental learning, tested in an LI+CTA protocol; see Materials and Methods), the ligands were applied shortly before the presentation of a novel taste in a latent inhibition experiment, and their effect on the efficacy of that same taste as a conditioned taste in subsequent CTA was determined by measuring CTA memory $3 \mathrm{~d}$ after CTA training. In the second type of protocol (CTA protocol), the ligands were applied shortly before the presentation of a novel taste in CTA training, and their effect on CTA memory was determined $3 \mathrm{~d}$ later. The two protocols partially overlap yet complement each other. The CTA protocol assesses the capability of a taste to become associated shortly after its consumption with a negative reinforcer and of the taste aversion association to be remembered in the long term; the LI+CTA protocol further assesses the capability of a taste per se to be remembered in the long term. Specifically, in the LI+CTA proto$\mathrm{col}$, the ligands were microinjected bilaterally $20 \mathrm{~min}$ before the preexposure to the novel taste $(10 \mathrm{ml}$ of $0.1 \%$ saccharin during 10 min). On the day of CTA training, $3 \mathrm{~d}$ after the LI preexposure, the animals were exposed for the second time to the same taste, and $\mathrm{LiCl}$ injected intraperitoneally was followed $40 \mathrm{~min}$ after the offset of drinking. Animals were tested 3 d later.

The following ligands were each microinjected into the IC: the NMDA receptor antagonist APV, the metabotropic glutamate receptor (mGluR) antagonist $\mathrm{MCPG}$, the $\mathrm{AMPA} /$ kainate receptor 
(AMPA/KR) antagonist NBQX, the muscarinic antagonist scopolamine, the GABAergic antagonist bicuculline, the GABAergic agonist muscimol, the $\beta$-adrenergic antagonist propranolol, and the dopaminergic $\mathrm{D}_{1} / \mathrm{D}_{5}$ antagonist $\mathrm{SCH}$. We found out that local microinjection to the IC of antagonists to the aforementioned glutamatergic receptors, muscarinic acetylcholine receptor, GABA receptors, dopamine $\left(D_{1} / D_{5}\right)$ receptors, and $\beta$-adrenergic receptors, as well as of the GABAergic agonist, impaired the encoding of taste memory as assessed in the LI+CTA protocol (Fig. 2A). As can be seen in the figure, the ligands were all able to abolish essentially all of the attenuating effect of LI on subsequent CTA training to the same taste. In the CTA protocol, all of these ligands attenuated CTA training when microinjected $20 \mathrm{~min}$ before the exposure to saccharin during conditioning (Fig. 2B). The aversion index of control rats injected intraperitoneally with saline instead of $\mathrm{LiCl}$ in a CTA protocol was $40 \pm 5$, whereas that of rats injected intraperitoneally with $\mathrm{LiCl}$ in the CTA protocol (and microinjected into the brain with vehicle only) was $96 \pm 3$. The ligands used in this study were able to diminish CTA by $38 \pm 6 \%$. This corresponds to the magnitude of blocking of long-term CTA by LI (Fig. $2 A$ ), as well as by other types of pharmacological inhibition of IC function (Rosenblum et al., 1993; Berman et al., 1998). It might represent a ceiling effect, unveiling the maximal contribution of the IC to the overall CTA behavior in our training and testing protocol. Indeed, the magnitude of blocking was not further augmented by coinjection of APV plus scopolamine into the cortex (data not shown).

\section{The drugs microinjected into the IC did not serve as reinforcers in CTA}

We were concerned with the possibility that the drugs microinjected into the IC might themselves induce visceral malaise, because a variety of drugs, including neurotransmitter antagonists, may induce malaise when administered systemically (MacMahon et al., 1981; Asin and Montana, 1989; Bures and Buresova, 1989). Although the drugs in our study were locally microinjected into the IC, and, moreover, this was done before the taste was presented, i.e., in a backward conditioning situation that is not expected to lead to efficient associations, we could not exclude the possibility that subtle malaise might develop that lingers throughout and after the time during which the tastant is consumed and become associated with the experienced taste. Furthermore, association of druginduced malaise with taste may under appropriate conditions overshadow the association of $\mathrm{LiCl}$ with the taste in the CTA protocol. Therefore, we have monitored the amount of saccharin consumed by rats that had been microinjected with each of the ligands $20 \mathrm{~min}$ before the onset of drinking, in the absence of $\mathrm{LiCl}$ intraperitoneal injection $40 \mathrm{~min}$ after the offset of drinking (Naor and Dudai, 1996), and compared it with the amount of saccharin consumed in a first re-presentation of the tastant $3 \mathrm{~d}$ later. If microinjection of a specific drug into the IC produced malaise, we would expect consumption of saccharin on its re-presentation $3 \mathrm{~d}$ later to be lower. This was not the case. The values for the control (vehicle microinjected into the IC) were $8.9 \pm 0.5$ versus $8.6 \pm 0.4 \mathrm{ml}$, respectively. The corresponding values for scopolamine, propranolol, $\mathrm{SCH}, \mathrm{APV}, \mathrm{MCPG}, \mathrm{NBQX}$, and bicuculline were $9.0 \pm 0.4$ versus $9.2 \pm 0.5,9.1 \pm 0.5$ versus $8.8 \pm 0.8,9.0 \pm 0.9$ versus $8.9 \pm 0.9$, $9.1 \pm 0.6$ versus $9.4 \pm 0.8,8.8 \pm 0.3$ versus $8.9 \pm 0.5,9.2 \pm 0.5$ versus $9.0 \pm 0.3$, and $8.9 \pm 0.9$ versus $9.5 \pm 1.0$. Hence, we did not detect any evidence for reinforcing properties of the ligands used when microinjected into the IC under the conditions used by us in this study.

\section{AMPA/KR antagonist and GABA receptor agonist impaired both acquisition and retrieval of taste memory}

The effect of a drug administrated during training on performance in a CTA memory test may be attributable to effects either on acquisition or on sensory or motor faculties essential for the behavioral performance of the task. In the latter case, the performance in a retrieval test is also expected to be impaired. To test the
A

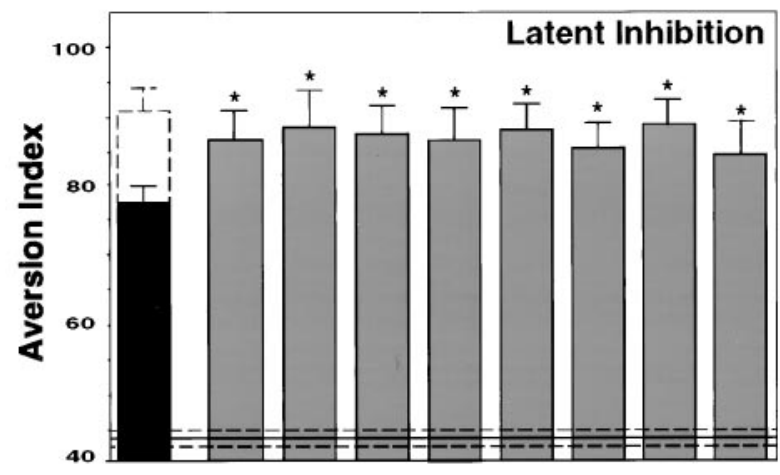

B
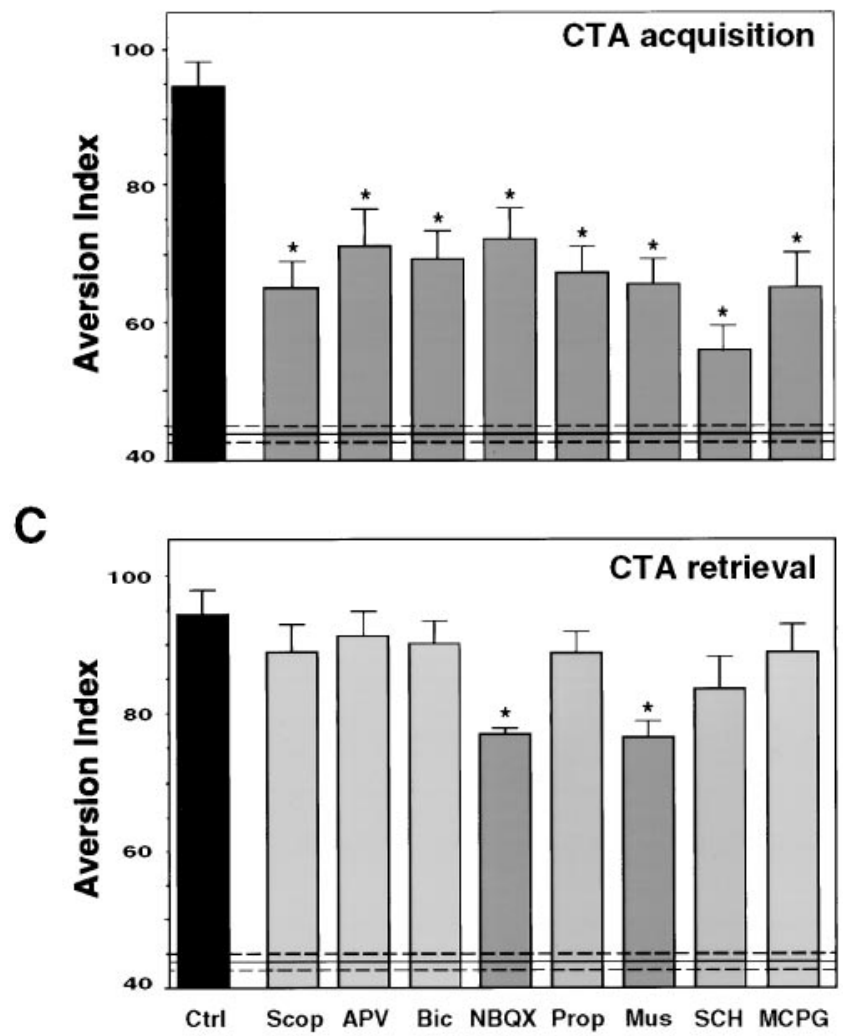

Figure 2. Behavioral effects of the different neurotransmitter ligands microinjected into the IC. $A$, Impairment of latent inhibition by local bilateral microinjection of the cholinergic antagonist scopolamine (Scop; $50 \mu \mathrm{g})$, the NMDA receptor antagonist APV $(10 \mu \mathrm{g})$, the GABAergic antagonist bicuculline $($ Bic $; 20 \mu \mathrm{g})$, the AMPA/kainate receptor antagonist NBQX (5 $\mu \mathrm{g})$, the $\beta$-adrenergic receptor antagonist propranolol (Prop; $20 \mu \mathrm{g}$ ), the GABAergic agonist muscimol (Mus; $5 \mu \mathrm{g}$ ), the $\mathrm{D}_{1} / \mathrm{D}_{5}$ dopamine receptors antagonist SCH $23390(S C H ; 5 \mu \mathrm{g})$, and the metabotropic glutamate receptor antagonist MCPG $(50 \mu \mathrm{g})$. Animals were trained and tested in the LI+CTA protocol as described in Materials and Methods. They were microinjected with the different ligands $20 \mathrm{~min}$ before the preexposure to the novel taste in the LI phase of the protocol $(n=6$ per group; the control black bar indicates aversion index value for CTA $+\mathrm{LI}$, and the dashed bar indicates CTA without LI). $B$, Effect of the ligands on CTA acquisition. Animals were microinjected $20 \mathrm{~min}$ before CTA training $(n=10)$ and tested as detailed in Materials and Methods in the CTA protocol. $C$, Effect of the ligands on CTA retrieval. Animals were trained and microinjected with the drugs $20 \mathrm{~min}$ before the test session in the CTA protocol, $3 \mathrm{~d}$ after the conditioning $(n=11)$. Aversion Index is defined as [milliliters of water/(milliliters of water + milliliters of saccharin $) \times 100]$. Injection volume is $1 \mu \mathrm{l} /$ hemisphere. An aversion index of 50 indicates equalpreference level. Ctrl, Control animals microinjected into the IC with vehicle only. Included for comparison is also the aversion index of shamconditioned controls, i.e., rats injected in training with saline intraperitoneally instead of $\mathrm{LiCl}$ intraperitoneally, and microinjected into the IC with vehicle only (43 \pm 3 , horizontal solid line \pm dashed lines $)$. 
A

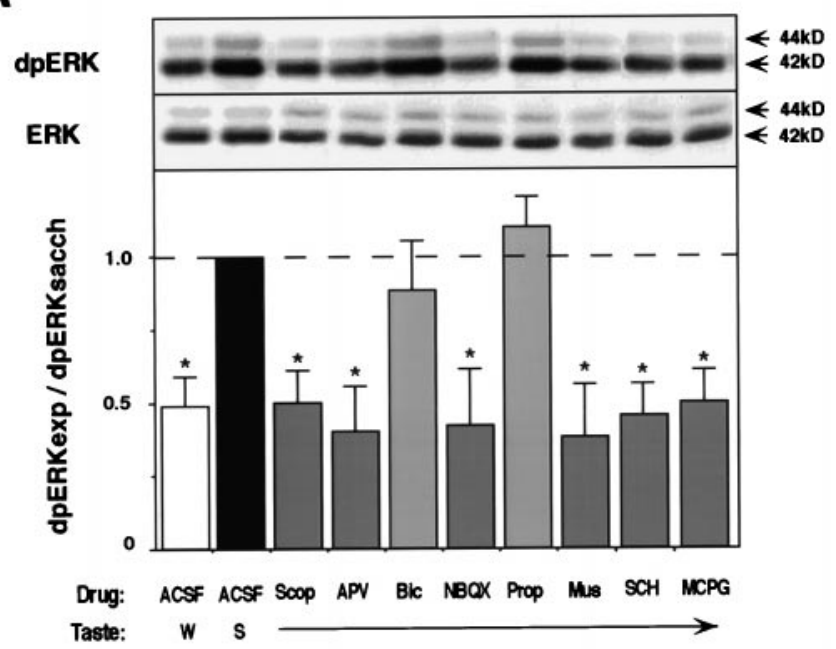

B

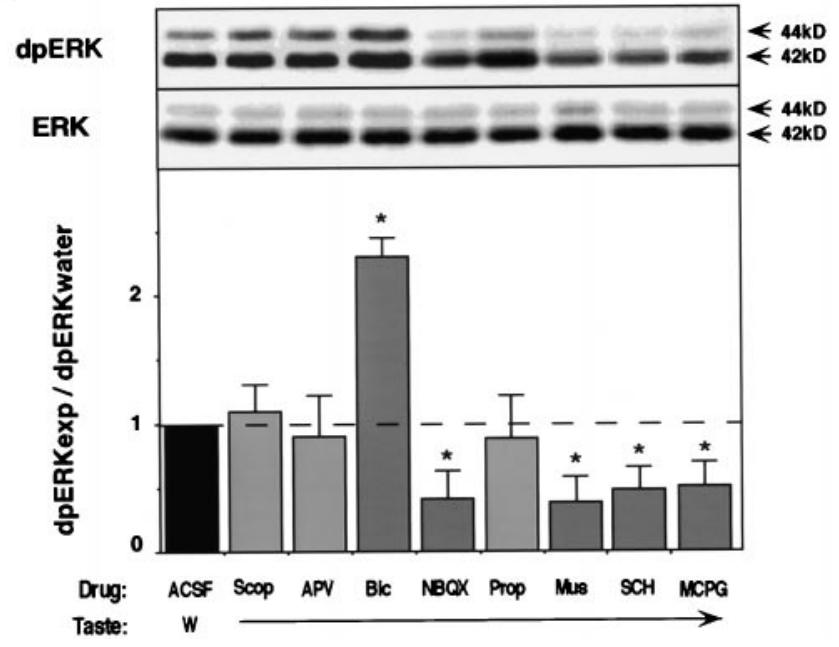

Figure 3. Effect of various ligands microinjected into the IC on ERK1-2 activation. $A$, Effect on taste-induced ERK1-2 activation. Top, representative blots of activated (dpERK1-2) and total ERK1-2 (ERK) from animals microinjected with the different neurotransmitter ligands $20 \mathrm{~min}$ before exposure to saccharin (black and gray bars) and water (white bar). Bottom, Quantification of the results from the blots $(n=10$ per group). The level of dpERK1-2 in animals microinjected with ACSF and exposed to saccharin (black bar) is used as standard. Ratio of activation is presented as dpERK experimental/dpERK saccharin. $B$, Effect of the ligands on the basal level of ERK1-2 activation. Top, Representative blots of dpERK1-2 and ERK1-2 from animals microinjected with the ligands and exposed to water 20 min later. Bottom, Quantification of the results from the blots $(n=12)$. The level of dpERK1-2 in animals microinjected with ACSF and exposed to water (black bar) is used as standard. Ratio of activation is presented as dpERK experimental/dpERK water. $W$, Water; S, saccharin.

role of the different neurotransmitter-neuromodulator systems in retrieval, we microinjected the drugs $20 \mathrm{~min}$ before the test. CTA retrieval was only affected by the microinjection of NBQX and muscimol (Fig. 2C).

\section{Perturbation of cholinergic, glutamatergic, dopaminergic, and GABAergic receptor function blocked taste-induced MAPK activation in the IC}

As shown previously, drinking an unfamiliar taste specifically and differentially activates ERK1-2 in the insular cortex of the behaving rat (Berman et al., 1998). To test the effect of the different neurotransmitter ligands on taste-induced ERK1-2 kinase activation, animals were microinjected with the antagonists into the IC 20 min before the exposure to the novel taste. Thirty minutes after the offset of drinking, the time in which ERK activation is maximal (Berman et al., 1998), the IC was excised, homogenized, and immunoblotted with antibodies that specifically recognize the double-phosphorylated, activated form of ERK1-2. As can be seen in Figure $3 A$, taste-induced MAPK activation was blocked by the microinjection of scopolamine, APV, MCPG, NBQX, SCH, and muscimol. The total amount of ERK protein remained unaltered in all treatments.

\section{The basal level of activation of ERK1-2 in the IC depends on the AMPA, mGluR, dopaminergic, and GABAergic receptors}

Which neurotransmitter system(s) is responsible for keeping the basal activity of MAPK in cortex? To answer this question, we microinjected the antagonists into the IC 20 min before drinking a familiar taste (tap water). The IC was then excised and treated as above. Only the microinjection of NBQX, MCPG, SCH 23390, and muscimol decreased the basal level of MAPK activation, whereas bicuculline increased it (Fig. 3B). No changes were observed in the total amount of ERKs.

\section{DISCUSSION}

When a rat samples an unfamiliar foodstuff, but not when it consumes a familiar one, molecular mechanisms are mobilized in the insular cortex, which probably encode the saliency of the event on the one hand and trigger long-term memory of the new taste on the other. Among these is activation of the ERK1-2 cascade, culminating in modulation of gene expression in cortical neurons (Berman et al., 1998). The activation of ERK1-2 attains maximal values within $30 \mathrm{~min}$ and subsides to "resting level" within $60 \mathrm{~min}$. It is not attributable merely to the perception of the taste; reacting to the same taste on a subsequent presentation, when it is already familiar, does not affect ERK1-2 activity. Hence, information in addition to that encoded in the sheer sensory percept must instruct the cortex that the taste is novel and activate ERK1-2. Furthermore, rats react to taste within seconds or even less (Halpern and Tapper, 1971), whereas the aforementioned molecular manifestation of novelty detection displays a kinetics with a $\tau_{1 / 2}$ of many minutes. It is therefore of interest to identify the pathways upstream of the ERK1-2 cascade that inform the cortical circuits about the novelty of the sensory input. The objective of the work presented in this paper was to contribute toward the identification of such pathways, as well as of their contribution to the formation of long-term memory of the new taste for which the activation of ERK1-2 is obligatory. This was done by introducing into the insular cortex, shortly before the consumption of a new taste, specific antagonists or agonists of identified neurotransmitter and neuromodulator systems and testing their effect on the activation of ERK1-2 and taste memory.

Our results show that, although multiple neurotransmitter systems in the IC are necessary for incidental taste learning and CTA learning, they do differ in the specificity of their effect on memory (Fig. $2 A, B$ ). Glutamatergic (AMPA, NMDA, mGlu), $\beta$-adrenergic, cholinergic (muscarinic), dopaminergic $\left(\mathrm{D}_{1}, \mathrm{D}_{5}\right)$, and $\mathrm{GABA}_{\mathrm{A}}$ receptors are all required for the acquisition of taste information. However, the AMPA and the GABAergic systems are also obligatory for retrieval of taste information, turning it likely that they play a role either in both acquisition and retrieval, or, more likely, also in other processes related to the perception of taste and the reaction to it. The results also show that the activation of ERK1-2 in the insular cortex by unfamiliar taste is specifically dependent on the activation of muscarinic acetylcholine receptors (mAChRs) and NMDAR (Fig. 3A,B).

Furthermore, our data indicate that the activation level of ERK1-2 in vivo in cortex is itself modulated in the resting level by a glutamatergic, dopaminergic, and GABAergic balance; in contrast, the muscarinic and $\beta$-adrenergic input, as well as glutamatergic input mediated via the NMDA receptor system, do not affect the basal level of ERK activation in the IC. The activity-dependent regulation of MAPKs in the nervous system has been investigated 


\section{Basal}

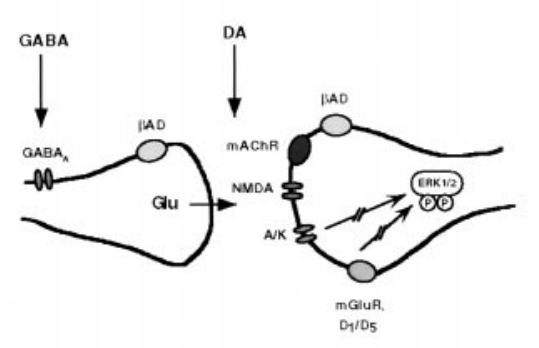

Acquisition

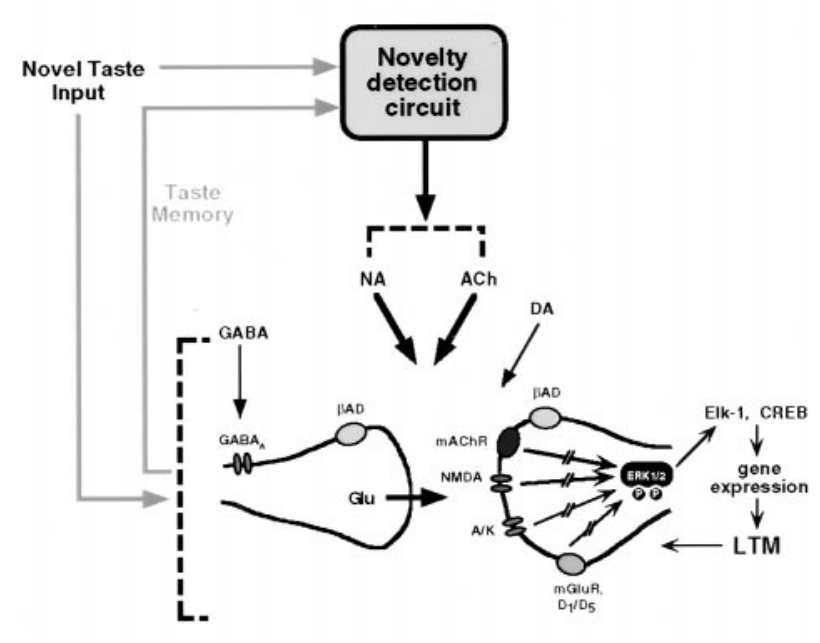

Retrieval

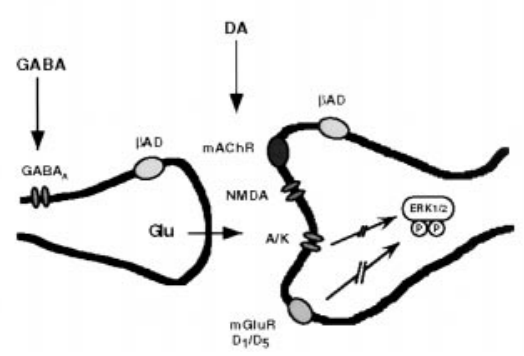

Figure 4. A highly simplified scheme of some elements in the processes that might subserve the encoding of taste memory in the IC. In the absence of taste input (Basal), the balance between the resting levels of glutamate (Glu; acting via AMPA/KR and mGluR), GABA, and dopamine ( $D A$; via $\mathrm{D}_{1} / \mathrm{D}_{5}$ receptors) regulates the basal level of ERK1-2 activation. When a new taste is consumed, a hypothetical novelty detection system, e.g., thalamocortico-brainstem circuits (Ahissar et al., 1997, Ahissar, 1998), compares the on-line stimulus with taste representations in memory. According to this model, if a meaningful mismatch is detected, a signal is sent to the cholinergic and noradrenergic systems, resulting in release of acetylcholine and noradrenaline in the IC. Acquisition involves, in addition to glutamatergic transmission via the metabotropic and AMPA receptors, glutamatergic transmission via the NMDA receptor, as well as cholinergic and noradrenergic input. Acetylcholine activates ERK1-2, whereas noradrenaline functions in this system independent of ERK1-2. Activation of ERK1-2 culminates in modulation of gene expression and ultimately in long-term representational changes. The yet unidentified novelty detection circuit may overlap with the IC circuits, which are altered by taste experience, and may also involve brain areas not mentioned in the scheme, e.g., amygdala (Schafe and Bernstein, 1996; Escobar and Bermudez-Rattoni, $2000)$. In retrieval, glutamatergic transmission that is essential for activation of the memory circuits and expression of the recalled behavior is mediated in the now-modified synapse (bold contours) via the AMPA/KR, whereas the NMDA, muscarinic, and noradrenaline receptors are no more obliga- in several types of preparations (English and Sweatt, 1996; Atkins et al., 1998; Berman et al., 1998; Kaminska et al., 1999; Robertson et al., 1999; Winder et al., 1999; Rosenblum et al., 2000), but only little is known on the involvement of identified neuromodulatory and neurotransmitter systems in MAPK activation in identified brain regions in the context of memory formation in the behaving organism. Atkins et al. (1998) reported that systemic administration of the NMDA receptor channel blocker MK-801 attenuated hippocampal MAPK activation during fear conditioning in the rat. Our results on the role of metabotropic glutamate receptors, muscarinic receptors, and dopamine receptors in MAPK activation in the IC are in agreement with those of Robertson et al. (1999) in hippocampal slices. However, we did not observe coupling of the $\beta$-adrenergic receptors to ERK1-2 activation in IC, as reported in the hippocampus by Robertson et al. (1999) and Winder et al. (1999). In cultured mammalian neurons, activation of ERKs was reported to be regulated by membrane depolarization and calcium influx (Rosen et al., 1994), G-protein-coupled receptors (Sudgen and Clerk, 1997; Lopez-Ilasca, 1998), and AMPA receptors (Hayashi et al., 1999). ERKs were also found to be modulated by GABA in immature cerebral granule cells (Fiszman et al., 1999) and by dopamine and glutamate in hippocampal slices (Otani et al., 1999) and in rat cortical neurons (Wang and Durkin, 1995; Das et al., 1997; Vincent et al., 1998). As to the involvement of intracellular signaling cascades, Robertson et al. (1999) suggested that dopaminergic and $\beta$-adrenergic modulation of MAPK takes place via the cAMP-dependent protein kinase signaling pathway, whereas muscarinic, glutamatergic-metabotropic, NMDA, and AMPA-mediated regulation of MAPK involves multiple types of calcium-mediated signaling. Rosenblum et al. (2000) have reported recently that, in primary cortical cultures and in the COS-7 model system expressing muscarinic receptor subtypes, the muscarinic agonist carbachol activates ERKs in Src-, phosphoinositide-3 kinase-, and calcium- dependent mechanisms; furthermore, on the basis of their own as well as earlier data they proposed that MAPK is an intracellular "coincidence detector" of information mediated by cholinergic and glutamatergic inputs. All in all, the available data indicate that there is no shortage of potential intracellular signaling mechanisms that could mediate the effect of the neurotransmitter and neuromodulators on ERK1-2 activation observed by us in the IC; however, in the present study, we focused only on the intercellular signaling involved in ERK1-2 activation, in its potential role in memory formation.

A major question is, how are the neuromodulatory systems such as the cholinergic and the noradrenergic systems informed that the taste input is novel? Novelty detection is expected to require some type of fast internal comparator that matches the on-line (sensory) information with off-line (memory) information and triggers an appropriate cascade of events only if a mismatch is detected. In our case, the process includes recruitment of neuromodulatory pathways, e.g., cholinergic, resulting in activation of ERK1-2, subsequent modulation of gene expression, and ultimately long-term alterations in the cortical circuits that encode the new information. A potential candidate for a fast internal comparator is a corticothalamo-brainstem system, which may share properties with the model proposed by Ahissar (Ahissar et al., 1997; Ahissar, 1998) for the mammalian somatosensory system. Hence, the thalamus may compare on-line sensory information encoded in the brainstem with taste memory representations retained in the $\mathrm{IC}$, and when a mismatch is identified, trigger behavioral response on the one hand, and

$\leftarrow$

tory. ERK activation remains unaltered by the retrieved information; its resting activity level is still regulated as in Basal above. The scenario in which some of the effects of the indicated neurotransmitters and neuromodulators on MAPK are mediated via interneurons that ultimately use different transmitters is omitted for the sake of simplicity. For further details, see Results. $A / K$, AMPA/KR; $\beta A D, \beta$-adrenergic receptor; $L T M$, long-term memory; $P P$, biphosphorylated, hence activated, ERK1-2. 
initiate memory encoding in the IC on the other. An additional, potentially complementary circuit may involve the amygdala. It interconnects with both the IC and the brainstem and fits to compare the new with the familiar and to signal the saliency of the novel situation (Lamprecht and Dudai, 2000). The amygdala was shown recently to be capable of modulating the ability of the IC to form taste memory (Escobar and Bermudez-Rattoni, 2000) and is involved in the cholinergic modulation of that memory (Gutierrez et al., 1999). The novelty-induced activation of ERK1-2 detected by us, is, according to this type of model, a mechanism downstream of the neuromodulatory input, which itself is downstream of the on-line-off-line mismatch detection that is triggered by the novel taste perception. It is plausible to assume that glutamatergic input encodes taste information in the IC, whereas multiple neuromodulatory systems are engaged by the "novelty detection circuits." Among these neuromodulatory systems, the cholinergic input activates ERK1-2, whereas the $\beta$-adrenergic system functions independent of the ERK1-2 cascade. Acetylcholine, dopamine, GABA, and glutamate are released by activity in the IC (Lopez-Garcia et al., 1990). Cholinergic input has been shown to play an obligatory role in the encoding of taste aversion memory (Naor and Dudai, 1996; Gutierrez et al., 1999). Noradrenaline was implicated in the encoding and consolidation of other types of emotionally charged memories (Crow and Wendlandt, 1976; Cahill et al., 1994; Przybyslawski et al., 1999).

A highly simplified scheme compiling the available data on the potential involvement of identified neurotransmitters and neuromodulators in the modification by novel taste experience of IC synapses is presented in Figure 4. Many questions remain open, such as which molecular mechanisms are activated by the noradrenergic input, or, more generally, what is the default response of the brain to taste perception. Is every tastant considered unfamiliar unless proven otherwise? In other words, are the neuromodulatory systems that trigger memory formation in the IC, all or part, activated by the identification of taste unfamiliarity or inhibited by the recognition of taste familiarity? The elucidation of these and similar questions requires analysis of the cellular response to familiar and unfamiliar tastes in cortex, thalamus, brainstem, and possibly other stations in the central taste circuits, such as amygdala. The identification of neurotransmitters and neuromodulators that are obligatory for the response to novelty and the encoding of taste memory should contribute to our understanding of the interaction and function of the circuits involved.

\section{REFERENCES}

Ahissar E (1998) Temporal-code to rate-code conversion by neuronal phase-locked loops. Neural Comput 10:597-650.

Ahissar E, Haidarliu S, Zacksenhouse M (1997) Decoding temporally encoded sensory input by cortical oscillations and thalamic phase comparators. Proc Natl Acad Sci USA 94:11633-11638.

Asin KE, Montana WE (1989) Studies on D1 and D2 dopamine receptor involvement in conditioned taste aversions. Pharmacol Biochem Behav 32:1033-1041.

Atkins CM, Selcher JC, Petraitis JJ, Trzaskos JM, Sweatt JD (1998) The MAPK cascade is required for mammalian associative learning. Nat Neurosci 1:602-609.

Berman DE, Hazvi S, Rosenblum K, Seger R, Dudai Y (1998) Specific and differential activation of mitogen-activated protein kinase cascades by unfamiliar taste in the insular cortex of the behaving rat. J Neurosci 18:10037-10044.

Bures J, Buresova O (1989) Conditioned taste aversion elicited by intracerebral administration of drugs. Acta Physiol Hung 74:77-93.

Bures J, Buresova O, Krivanek J (1988) Brain and behavior: paradigms for research in neural mechanisms. New York: Wiley.

Bures J, Bermudez-Rattoni F, Yamamoto T (1998) Conditioned taste aversion. Memory of a special kind. Oxford: Oxford UP.

Burnette WW (1981) Western blotting: electrophoretic transfer of proteins from sodium dodecyl sulfate-polyacrylamide gels to unmodified nitrocellulose and radiographic detection with antibody and radioiodinates protein A. Anal Biochem 112:195-203.

Cahill L, Prins B, Weber M, McGaugh JL (1994) $\beta$ adrenergic activation and memory for emotional events. Nature 371:701-704.

Crow JT, Wendlandt S (1976) Impaired acquisition of a passive avoidance response after lesions induced in the locus coeruleus by 6-OH-dopamine. Nature 259:42-44.
Das S, Grunert M, Williams L, Vincent SR (1997) NMDA and $\mathrm{D}_{1}$ receptors regulate the phosphorylation of CREB and the induction of c-fos in striatal neurons in primary culture. Synapse 25:227-233.

English JD, Sweatt JD (1996) Activation of p42 mitogen-activated protein kinase in hippocampal long term potentiation. J Biol Chem 271:24329-24332.

Escobar ML, Bermudez-Rattoni F (2000) Long-term potentiation in the insular cortex enhances conditioned taste aversion retention. Brain Res 852:208-212.

Fiszman ML, Borodinsky LN, Neale JH (1999) GABA indices proliferation of immature cerebellar granule cells grown in vitro. Dev Brain Res 115:1-8.

Gutierrez H, Gutierrez R, Ramirez-Trejo L, Silva-Gandarias R, Ormsby CE, Miranda MI, Bermudez-Rattoni F (1999) Redundant basal forebrain modulation in taste aversion memory formation. J Neurosci 19:7661-7669.

Halpern BP, Tapper DN (1971) Taste stimuli: quality coding time. Science 171:1256-1258.

Hayashi T, Umemori H, Mishina M, Yamamoto T (1999) The AMPA receptors interacts with and signals through the protein tyrosine kinase fyn. Nature 397:72-76.

Kaminska B, Kaczmarek L, Zangenehpour S, Chaudhuri A (1999) Rapid phosphorylation of Elk-1 transcription factor and activation of MAP kinase signal transduction pathways in response to visual stimulation. Mol Cell Neurosci 13:405-414.

Kiefer SW, Braun JJ (1977) Absence of differential associative responses to novel and familiar taste stimuli in rats lacking gustatory cortex. J Comp Physiol Psychol 91:498-507.

Kosar E, Grill HJ, Norgren R (1986) Gustatory cortex in the rat. I. Physiological properties and cytoarchitecture. Brain Res 379:329-341.

Laemmli UK (1970) Cleavage of structural proteins during the assembly of the head of bacteriophage $\mathrm{T}_{4}$. Nature 369:156-160.

Lamprecht R, Dudai Y (2000) Conditioned taste aversion and the amygdala: it's there, but where? In: The amygdala (Aggleton J, ed). Oxford: Oxford UP, in press.

Lopez-Garcia JC, Bermudez-Rattoni F, Tapia R (1990) Release of acetylcholine, $\gamma$-aminobutyrate, dopamine and glutamate, and activity of some related enzymes, in rat gustatory neocortex. Brain Res 523:100-104.

Lopez-Ilasca M (1998) Signaling from G-protein coupled receptors to mitogen-activated protein (MAP)-kinase cascades. Biochem Pharmacol 56:269-277.

Lubow RE (1989) Latent inhibition and conditioned attention theory. London: Cambridge UP.

MacMahon SW, Blampied NM, Hughes RN (1981) Aversive stimulus properties of scopolamine. Pharmacol Biochem Behav 15:389-392.

Mohammed AK, Callenholm NEB, Jarbe TUC, Swedberg MDB, Danysz W, Robbins TW, Archer T (1986) Role of central noradrenaline neurons in the contextual control of latent inhibition in taste aversion learning. Behav Brain Res 21:109-118.

Naor C, Dudai Y (1996) Transient impairment of cholinergic function in the rat insular cortex disrupts the encoding of taste in conditioned taste aversion. Behav Brain Res 79:61-67.

Otani S, Auclair N, Desce JM, Roisin MP, Crepel F (1999) Dopamine receptors and groups I and II mGluRs cooperate for long-term depression induction in rat prefrontal cortex through converging postsynaptic activation of MAP kinases. J Neurosci 19:9788-9802.

Otawa S, Takagi K, Ogawa H (1995) NMDA and non-NMDA receptors mediate taste afferent inputs to cortical taste neurons in rats. Exp Brain Res 106:391-402.

Paxinos G, Watson C (1986) The rat brain in stereotaxic coordinates, Ed 2. New York: Academic.

Przybyslawski J, Roullet P, Sara SJ (1999) Attenuation of emotional and nonemotional memories after their reactivation: role of $\beta$ adrenergic receptors. J Neurosci 19:6623-6628.

Quillfeldt JA, Schmidtz PK, Walz R, Bianchin M, Zantta MS, Medina JH, Izquierdo I (1994) CNQX infused into entorhinal cortex blocks memory expression, and AMPA reverts the effect. Pharmacol Biochem Behav 48:437-440.

Revusky S, Garcia J (1970) Learned associations over long delays. Psychol Learn Motiv 4:3-84.

Riedel G (1996) Function of metabotropic glutamate receptors in learning and memory. Trends Neurosci 19:219-224.

Riedel G, Wetzel W, Reymann KG (1994) (R,S)-alpha-methyl-4carboxyphenylglycine (MCPG) blocks spatial learning in rats and longterm potentiation in the dentate gyrus in vivo. Neurosci Lett 167:141-144.

Robertson ED, English JD, Paige Adams J, Selcher JC, Kondratick C, Sweatt JD (1999) The mitogen-activated protein kinase cascade couples PKA and PKC to cAMP response element binding protein phosphorylation in area CA1 of hippocampus. J Neurosci 19:4337-4348.

Rosen L, Ginty DD, Weber MJ, Greenberg ME (1994) Membrane depolarization and calcium influx stimulate MEK and MAP kinase via activation of Ras. Neuron 12:1207-1221.

Rosenblum K, Meiri N, Dudai Y (1993) Taste memory: the role of protein synthesis in gustatory cortex. Behav Neural Biol 59:49-56.

Rosenblum K, Berman DE, Hazvi S, Dudai Y (1996) Carbachol mimics effects of sensory input on tyrosine phosphorylation in cortex. NeuroReport 7:1401-1404. 
Rosenblum K, Berman DE, Hazvi S, Lamprecht R, Dudai Y (1997) NMDA receptor and the tyrosine phosphorylation of its $2 \mathrm{~B}$ subunit in taste learning in the rat insular cortex. J Neurosci 17:5129-5135.

Rosenblum K, Futter M, Jones M, Hulme EC, Bliss TVP (2000) ERK I/II regulation by the muscarinic acetylcholine receptors in neurons. J Neurosci 20:977-985.

Schafe GE, Bernstein IL (1996) Forebrain contribution to the induction of a brainstem correlate of conditioned taste aversion. I. The amygdala. Brain Res 741:109-116.

Schafe GE, Bernstein IL (1998) Forebrain contribution to the induction of a brainstem correlate of conditioned taste aversion. II. Insular (gustatory) cortex. Brain Res 800:40-47.

Sudgen PH, Clerk A (1997) Regulation of the ERK subgroup of MAPK kinase cascades through $G$ protein-coupled receptors. Cell Signaling 9:337-351.
Thorndike EL, Rock RT (1934) Learning without awareness of what is being learned or intent to learn it. J Exp Psychol 17:1-19.

Vincent SR, Sebben M, Dumuis A, Bockaert J (1998) Neurotransmitter regulation of MAP kinase signaling in striatal neurons in primary culture. Synapse 29:29-36.

Wang Y, Durkin JP (1995) $\alpha$-amino-3-hydroxy-5-methyl-4-isoxazolepropionicacid, but not $N$-methyl-D-aspartate, activated mitogen-activated protein kinase through G-protein $\beta \gamma$ subunits in rat cortical neurons. J Biol Chem 270:22783-22787.

Winder DG, Martin KC, Muzzio IA, Rohrer D, Chruscinski A, Koblika B, Kandel ER (1999) ERK plays a regulatory role in induction of LTP by theta frequency stimulation and its modulation by $\beta$-adrenergic receptors. Neuron 24:715-726. 